\title{
Francisco Javier Clavijero oder die amerikanische Antike
}

Wenn wir uns nun Francisco Javier Clavijero zuwenden, so tun wir dies im Bewusstsein der Tatsache, dass jede Geschichte eine Vision nicht nur der Gegenwart und ihrer möglichen Zukünfte, sondern ganz grundsätzlich auch der Vergangenheiten ist, die für ein bestimmtes Verständnis von Geschichte als grundlegend angesehen werden und damit Traditionen bilden, aus denen sich die Sicht der Gegenwart speist. Und mit dem Neuspanier Clavijero wenden wir uns zugleich einem streitbaren Geist zu, der in der bereits erwähnten „Disputa del Nuovo Mondo“ eine herausragende Rolle spielte und damit in den transatlantischen Auseinandersetzungen um die Neue Welt ein gewichtiges Wort bezüglich der Aufklärungsphilosophie mitzureden hatte. Dass die Lebensläufe neuspanischer Aufklärer nicht selten in den Bereich des Klerus führen und damit die Verwicklung in mancherlei innere Widersprüche verbunden ist, werden wir im Verlauf dieser Vorlesung noch wiederholt bemerken.

Francisco Javier Clavijero wurde am 9. September $1731 \mathrm{im}$ damals neuspanischen und heute mexikanischen Puerto de Veracruz, der wichtigen Hafenstadt an der Karibikküste, geboren und starb am 2. April 1787 im italienischen Bologna. Er war das dritte von insgesamt elf Kindern und kam durch seinen Vater, der im Auftrag der spanischen Krone das Landesinnere Neuspaniens und damit stark von den indigenen Kulturen geprägte Regionen durchzog, früh schon in Kontakt mit der indigenen Bevölkerung, ihren Sitten, Gebräuchen und Vorstellungen. Dieser Kontakt war für den künftigen Jesuiten grundlegend und erklärt, warum er sich früh schon Kenntnisse des Náhuatl aneignete, der Sprache der Mexica. Seine Ausbildung erfuhr der Junge im Colegio de San Jerónimo zu Puebla, das ebenso von den Jesuiten betrieben wurde wie das Colegio de San Ignacio, wo er seine Studien bei den Jesuiten fortsetzte. Er verfügte damit von Beginn an über einen weiten Bildungsghorizont, den er im weiteren Verlauf seines Lebens beständig ausbaute.

Im Jahre 1748 trat Clavijero im Colegio de Tepotzotlán dem Jesuitenorden bei, an dessen Disziplin er sich anfangs rieb. Er bereitete sich auf die Priesterweihe vor, fiel seinen Ordensbrüdern aber schnell als hochtalentierter junger Mann auf, der in seinen Lektüren unstillbar war. Rasch arbeitete er sich auch in das Gebiet der Philosophie ein und las die Arbeiten von René Descartes, Pierre Gassendi, Isaac Newton oder Gottfried Wilhelm Leibniz. Daneben studierte er aber auch das Náhuatl, dessen Erlernen für angehende Priester damals wohl obligatorisch war. In seinen Studien wandte er sich Carlos de Sigüenza y Góngora $\mathrm{zu}$, einem Spezialisten des 17. Jahrhunderts für indigene Kulturen, und beschäf- 
tigte sich verstärkt mit den schriftlichen Überlieferungen der altamerikanischen Kulturen. Daneben setzte er seine Lektüren ,moderner` europäischer Philosophen fort.

Auf Grund seines Talents und seiner Fähigkeiten unterrichtete er bald an den Colegios beziehungsweise Universitäten von Puebla, Morelia und Guadalajara, wobei er der Rhetorik und der ,modernen“ Philosophie seine besondere Aufmerksamkeit schenkte. Er verfasste in dieser Zeit mehrere Aufsätze in lateinischer Sprache, die zum größten Teil nicht mehr auf uns gekommen sind, in denen sich der Jesuit aber ebenso der griechischen Philosophie wie der zeitgenössischen Aufklärungsphilosophie Europas zuwandte.

1754 wurde er zum Priester geweiht; doch seinen Wünschen, in die Indianermissionen etwa nach Kalifornien versetzt zu werden, wurde nicht stattgegeben. 1758 kam er ins Colegio de San Gregorio in Mexiko-Stadt, wo er intensiv mit indigenen Schülern in Berührung kam und zugleich seine Studien altamerikanischer Kulturen fortsetzen konnte. Dabei griff er auch auf die Sammlungen von Carlos Sigüenza y Góngora zurück. Clavijero beschäftigte sich in dieser Zeit ausführlich mit den „Pinturas“, also mit indigenen Aufschreibesystemen, die ihm in den Archiven der Hauptstadt zur Verfügung standen.

Der noch junge Jesuit wurde, darin durchaus seinen Wünschen entsprechend, vorwiegend in der Unterweisung indianischer Schüler eingesetzt. In Mexiko-Stadt und anderswo übte er mit seinen Lehren und Vorstellungen einen starken Einfluss auf seine Schüler aus, zu denen - dann in Morelia - unter anderem Miguel Hidalgo zählte, eine der großen Figuren der mexikanischen Unabhängigkeit, der man auf Grund der von Hidalgo initiierten revolutionären Aktivitäten bis heute den Ehrentitel eines „Padre de la Patria“ gab. In Neuspanien wie anderswo in den Amerikas gab es eine Beziehung zwischen Aufklärung und Revolution, obwohl man mit direkten Verbindungen zwischen beiden vorsichtig umgehen muss. Auch andere Vertreter der neuspanischen Aufklärung wie der neuspanische Philosoph und Historiker José Antonio de Alzate y Ramírez zählten zu Clavijeros Schülern.

Dann aber erfolgte jener historische Einschnitt, der sein Leben in zwei Hälften teilte. Dieser Bruch begann 1767 mit dem Dekret des spanischen Königs Carlos III, das zur Vermeidung einer Unterwanderung staatlicher Strukturen durch die Gefolgsleute des Heiligen Ignatius von Loyola alle Jesuiten zum sofortigen Verlassen der spanischen Kolonien in Amerika zwang. Die Auswirkungen dieses Erlasses waren weitreichend, befand sich doch ein wesentlicher Teil des kolonialen Bildungswesens traditionell in jesuitischer Hand. Aus weiten Teilen des öffentlichen Lebens und der Verwaltung waren sie kaum noch wegzudenken - und ihre beherrschende Rolle in Paraguay war selbst den Leserinnen und Lesern von Voltaires Candide plakativ vor Augen geführt worden. Um das 
Verbot des reichen Ordens und die sofortige Ausweisung rankten sich zahlreiche Mythen, insofern man etwa die Jesuiten verdächtigte, ihre großen Schätze noch rechtzeitig vor dem Zugriff des Kolonialstaates in Sicherheit gebracht zu haben, so dass sich nachfolgende Generationen insbesondere im 19. Jahrhundert immer wieder darum bemühten, den vielberufenen „Schatz der Jesuiten“ aufzufinden.

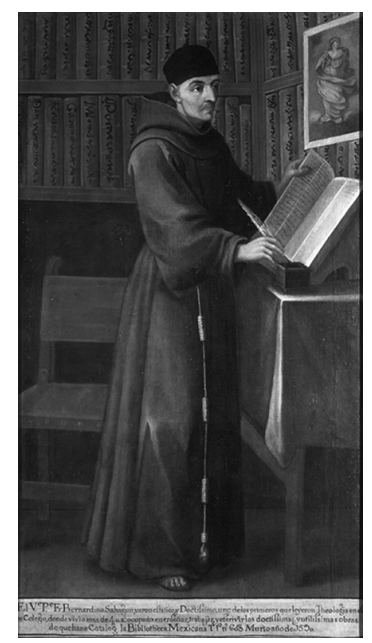

Abb. 28: Francisco Xavier Clavijero (1731-1787).

Mit der Ausweisung aller Jesuiten wurde über Nacht aus Clavijero ein Exilant. Er musste von Guadalajara aus das Nötigste zusammenpacken und über den Hafen von Veracruz zunächst im Oktober 1767 nach La Habana ausreisen. Schließlich führten in die Wege des Exils wie viele andere Jesuiten nach Bologna, das damals zum Kirchenstaat gehörte und wo er 1770 ankam. In den Folgejahren wurde Bologna bis zu Clavijeros Tod im Jahr 1787 der Ausgangspunkt all seines Denkens und Schreibens. Aus dem Leser, Lehrer und Denker wurde nun der Intellektuelle und Schriftsteller, als der er in die Geschichte einging.

Im italienischen Exil griff Francisco Javier Clavijero auf seine vielen Erinnerungen und Begegnungen mit indigenen Kulturen in Neuspanien, vor allem aber auf zahlreiche Dokumente in den Archiven des Kirchenstaates über die Geschichte der indianischen Kulturen zurück. In spanischer Sprache verfasste er sein historisches, philosophisches und anthropologisches Hauptwerk, seine berühmte Historia antigua de México, die 1780/81 zunächst auf Italienisch unter dem Titel Storia antica del Messico erschien. Erst 1826 wurde sein Werk in London in spanischer Sprache veröffentlicht; doch fand bereits die italienische Übersetzung in Europa eine nicht geringe Verbreitung und erregte Aufsehen. Dies war wichtig, denn Clavijero hatte bemerkt, wie falsch, abfällig und irre- 
führend sich im Jahrhundert der Aufklärung europäische Philosophen wie der Holländer Cornelius de Pauw oder der Schotte William Robertson über indigene Kulturen äußerten. Sie taten dies ohne jede eigene Kenntnis, waren nicht an Forschungen interessiert, lehnten alle zur Verfügung stehenden indigenen Quellen ab und entwarfen ein Bild der altamerikanischen Kulturen, das auf grausam eurozentrische Weise negativ entstellt war. Dagegen schrieb der neuspanische Jesuit nun von Europa aus an!

In seiner Historia antigua de México wandte sich Clavijero gegen alle europäischen Philosophen und Länder wie Frankreich, England, Österreich oder Preußen, in denen zumeist in französischer Sprache derartige Verunglimpfungen der indigenen Kulturen Amerikas erschienen waren. Clavijero kämpfte mit sachlichen, bisweilen aber auch polemisch eingefärbten Argumenten gegen jeden Versuch, die Kulturen Amerikas als barbarisch und alles Indigene als Barbarei abzutun. Wie schon der Titel seines Hauptwerkes suggerierte, unterstrich der Jesuit nicht nur die Gleichwertigkeit der indigenen Kulturen mit der europäischen Kulturentwicklung, sondern stellte die Welt des alten Amerika auf eine Stufe mit der griechisch-römischen Antike des Mittelmeerraumes. Wir werden auf die Argumente wie auf die Polemiken, die Clavijero anstieß und die sein Werk vorantrieb, sogleich detailliert eingehen.

Auch in seinem Dialog Filaletes y Paleófilo hatte der Jesuit zuvor schon seine Auffassung unterstrichen, dass die Wahrheit nicht bei den herrschenden Lehrmeinungen und den jeweils dominanten Doktrinen, sondern nur beim Studium der physischen Dinge und Realitäten gefunden werden könne. Diese Überzeugungen setzte er nun um: Entschieden sprach er sich für eine empirische Fundierung allen Wissens und für erfahrungsbasierte Wissenschaftskonzepte aus, welche nach eigenem Bekunden die Grundlage seines ganzen Schaffens bildeten.

Zugleich aber war er als Jesuit darum bemüht, das neu gefundene Wissen mit der christlichen Lehre zu verbinden. In Übereinstimmung mit seinem Orden glaubte er fest daran, dass die Erforschung alter Traditionen mit der ,modernen“ Philosophie des 17. und 18. Jahrhunderts ausgesöhnt werden könne und das Fundament für ein neues Wissen bieten würde, das es gegen alle oberflächlichen europäischen „Philosophes“ und deren vorgefasste Meinungen zu verteidigen gelte. ${ }^{1}$ Dass sich aus diesen Auffassungen Widersprüche zwischen der christlichen Lehre und der Aufklärungsphilosophie ergeben mussten, liegt auf

1 Vgl. zur philosophischen Einschätzung Clavijeros Krumpel, Heinz: Aufklärung und Romantik in Lateinamerika. Ein Beitrag zu Identität, Vergleich und Wechselwirkung zwischen lateinamerikanischen und europäischen Denkern. Frankfurt am Main - Berlin - New York: Peter Lang 2004. 
der Hand. Doch war es die große Leistung des neuspanischen Philosophen, auf Basis zahlreicher Quellen und Dokumente ein neues, deutlich ausgewogeneres Bild der altamerikanischen Kulturen entworfen zu haben, als die europäischen Aufklärungsphilosophen dies jemals entfaltet hatten. Zugleich weckte Clavijero Verständnis für die soziale Lage der indigenen Bevölkerung in den Kolonien, ein wichtiger Aspekt, der nicht zuletzt in die sozialrevolutionäre Seite der mexikanischen Unabhängigkeitsrevolution einging.

Francisco Javier Clavijero hatte mit seinem kulturhistorischen Werk die Vergangenheit Neuspaniens verändert, womit er zugleich eine neue Zukunft für Mexiko eröffnete. Gerade die kreolischen Trägerschichten der Unabhängigkeitsrevolution fassten Clavijeros Werk als wichtigen Impuls für ihr Aufbegehren gegen die spanische Kolonialherrschaft auf. Wir werden beim Dominikanermönch Fray Servando Teresa de Mier noch sehen, wie gerade die Neubewertung der Vergangenheit, aber auch die Verbindung von Aufklärung und christlichem Glauben zu einer der wesentlichen Grundlagen der Independencia werden konnte. Clavijeros neues Bild der altamerikanischen Kulturen stimulierte ein erneuertes amerikanisches Selbstbewusstsein, das sich nunmehr auf eine eigene große Vergangenheit, auf eine eigene Antike stützen konnte und auf dieser Grundlage neue Zukunftsmöglichkeiten entwarf.

In der seiner mehrbändigen Geschichte vorangestellten „Noticia de los escritores de la historia antigua de México“ listete der belesene Jesuit, der im Exil seine Kenntnisse weiter vertieft hatte, nicht etwa - wie dies ein europäischer Leser hätte erwarten können - die in Europa bekannten Autoritäten auf. Der kämpferische Geistliche, der weit davon entfernt war, sich wie viele Ordensbrüder in Italien einen gemächlichen Ruhestand $\mathrm{zu}$ verbringen, setzte gleich zu Beginn ein unübersehbares Zeichen. Er erweiterte in diesem seiner Historia antigua de México vorangestellten Text nicht nur die Basis schriftlicher Quellen, indem er eine große Zahl bislang weitgehend unbekannter, in Mexiko verfasster Texte einbezog, sondern auch indem er den Begriff von Schrift selbst radikal veränderte.

Dabei nahm er kein Blatt vor den Mund: Die von Antonello Gerbi so benannte Disputa del Nuovo Mondo trägt die Bezeichnung völlig zurecht. ${ }^{2}$ So warf er William Robertsons einflussreicher Geschichte Amerikas ${ }^{3}$ nicht nur vor, eine Vielzahl in alphabetischer Schrift vorliegender Texte übergangen und aus Unkenntnis ihre Existenz geleugnet $\mathrm{zu}$ haben; er unterstrich vielmehr mit Nach-

2 Vgl. das Standardwerk von Gerbi, Antonello: La Disputa del Nuovo Mondo. Storia di una polemica 1750-1900. Mailand - Neapel 1955.

3 Vgl. Robertson, William: The History of America. 2 Bde. London: W. Strahan 1777. 
druck, dass es nicht angehen könne, die indianischen Bilderhandschriften als unverständlich (beziehungsweise von unklarer Bedeutung, „de significación ambigua“) abzuqualifizieren.

Der Schotte habe, von europäischen Vorurteilen geleitet, sich nicht mit den indigenen Kulturen beschäftigt und auch keine nähere Kenntnis gehabt über jene Zeugnisse indianischer Schreibkunst, die Robertson längst zerstört geglaubt hatte. Francisco Javier Clavijero aber hielt dagegen:

Es gibt nicht wenige historische Malereien (pinturas), die der Inquisition der ersten Missionare entkamen, und sie wurden mit Respekt für den unsagbaren Überfluss, den es davon, wie man in meiner Geschichte sieht, zuvor gab, in jener von Torquemada und von anderen Schriftstellern erwähnt [...]. Weder sind diese Malereien von unklarer Bedeutung, außer für Robertson und für all jene, die nicht die Zeichen und die Figuren der Mexikaner verstehen, noch kennen sie das Verfahren, welches diese hatten, um die Dinge zu repräsentieren, so als wären unsere Schriften von unklarer Bedeutung für all jene, die eben nicht lesen können. Als man für die Missionare jene beklagenswerte Verbrennung der Malereien durchführte, lebten noch viele Geschichtsschreiber der Acolhuas, der Mexicas, der Tepaneken, der Tlaxkalteken usw., welche daran arbeiteten, den Verlust dieser Denkmäler wettzumachen, wie sie es zum Teil auch erreichten; denn sie machten neue Malereien oder bedienten sich unserer Zeichen, die sie bereits erlernt hatten, oder unterrichteten mündlich ihre eigenen Prediger in ihren Altertümern, und auf diese Weise konnten sie diese in ihren Schriften bewahren, ganz wie dies Motolinia, Olmos und Sahagún taten. ${ }^{4}$

Von Beginn seiner mehrbändigen Historia an lässt Clavijero keinen Zweifel daran, dass er nicht nur die Alphabetschrift, sondern auch andere Schreibverfahren als Schrift und zugleich Ausdruck einer verehrenswerten Kultur ansieht. Wichtig ist ferner, dass ebenso die Schrift wie die Schreiber, ebenso die Tradition wie die Kultur in ihrer Gesamtheit mit jener des Abendlandes auf ein und dieselbe Stufe gestellt werden. Wer die Texte dieser altamerikanischen Kulturen nicht zu lesen vermag, verhält sich nicht anders als einer, der des Lesens der Alphabetschrift nicht kundig ist, sich gleichwohl aber ein vernichtendes Urteil darüber erlaubt. Damit stellt Clavijero explizit die Kulturtechnik der ihm bekannten Bilderhandschriften aztekischer, tlaxcaltekischer oder anderer Herkunft gleichrangig neben die Kulturtechnik der Alphabetschrift, derer sich im Übrigen auch indianische Autoren bedient hatten. So erweitert der neuspanische Geschichtsschreiber die Quellenbasis, indem er die nicht weniger eindeutige Lesbarkeit derartiger Pinturas unterstreicht: Wer etwas Ernstzunehmendes

4 Clavijero, Francisco Javier: Historia antigua de México. Prólogo de Mariano Cuevas. Edición del original escrito en castellano por el autor. México, D.F.: Editorial Porrúa 1982, S. xxxiv. 
über diese Kulturen aussagen möchte, der muss schon deren grundlegende Kulturtechniken beherrschen!

Dies ist eine Kritik, die nicht nur für die vielen selbsternannten Kulturkritiker Altamerikas vernichtend ausfällt; sie trägt auch deutlich den Stempel der Aufklärung - freilich einer Aufklärung, die aus Amerika kommt. Der in dieser „Noticia“ aufgespannte explizite literarische Raum, der durch die ausdrückliche Erwähnung von und Bezugnahme auf literarische beziehungsweise naturhistorische Schriften und Dokumente gebildet wird, ist unverkennbar als Gegenraum zu jenem der europäischen „Philosophes“ konstruiert. Der gebildete und an den Konventionen seiner Zeit orientierte Jesuit konnte es jedoch schon mit Rücksicht auf seine Autorität bei einem traditionell an Europa orientierten Lesepublikum nicht unterlassen, seine Leserschaft darauf aufmerksam zu machen, dass er ungeachtet der großen Kosten, die die Literaturbeschaffung von Europa aus verursacht habe, alles gelesen habe, was zum Thema der altamerikanischen Kulturen veröffentlicht worden sei. Dabei unterstrich er einmal mehr ganz wie Raynal und in Befolgung der diskursiven Normen seiner Zeit - seine quellenkritische Haltung:

\footnotetext{
Ich habe aufmerksam alles gelesen und untersucht, was bis zum gegenwärtigen Zeitpunkt über diese Materie veröffentlicht wurde; ich habe die Berichte der verschiedenen Autoren miteinander konfrontiert und ihre Autorität auf der Waage der Kritik gewogen; ich habe sehr viele historische Malereien der Mexica studiert; ich habe mich dabei ihrer Manuskripte bedient, welche ich damals las, als ich noch in Mexiko weilte, und viele erfahrene Männer aus jenen Ländern konsultiert. ${ }^{5}$
}

In konzentrierter Form macht Clavijero in diesen Zeilen klar, dass er sich mit den altamerikanischen Kulturen nicht nur mit Hilfe von Büchern in europäischen Bibliotheken beschäftigte, sondern konkrete Feldforschung vor Ort in Neuspanien betrieb, sich viele Manuskripte ansah und mit Experten beriet.

Auch wenn wir uns hier der in epistemologischer Hinsicht wichtigen Tatsache nicht länger zuwenden können, dass der Autor in der Folge ebenso auf die Befragung von Schrifttexten und Informanten wie auch auf seine sechsunddreißigjährige Lebenserfahrung in verschiedenen Landesteilen (,provincias“) Mexicos hinweist, so bleibt doch festzuhalten, dass der so ausgespannte literarische Raum zugleich wesentlich breiter angelegt und pluri-kulturell zusammengesetzt ist. Mit anderen Worten: Bei der Behandlung der indigenen Kulturen der Vergangenheit sprengt der neuspanische Jesuit den Alphabet-schriftlichen Raum und eröffnet der Forschung wie der Aufklärungsphilosophie zu den Kulturen Amerikas zuvor nur selten befragte Bereiche nicht-europäischer Schriftsysteme.

5 Ebda., S. xxii. 
Der Raum des ,Eigenen“ ist für den amerikanischen Autor ein gegenüber dem der europäischen Autoren anderer; und er ist vor allem mehr, umfasst er doch auch das europäisch-okzidentale Schrifttum, das Clavijero als Jesuit ebenso im Bereich der christlichen Religion vertrat. Dass dies eine Distanzierung von den Verbrennungen indigener Texte und Manuskripte durch die (keineswegs nur frühneuzeitlichen) Kirchenoberen mit einschließt, versteht sich von selbst.

Francisco Javier Clavijero verfügte vor der Ausweisung der Jesuiten im Jahre 1767 über die Möglichkeit, die besten Bibliotheken und Archive des Landes wie auch gelehrte Vertreter der Universität von Mexico konsultieren zu können, der er seine Historia antigua de México auch zueignete ${ }^{6}$. Die Vertreter dessen, was man damals unter ,moderner' Philosophie aus Europa verstand, waren ganz selbstverständlich in seine Forschungen miteinbezogen. Descartes, Leibniz, Buffon, Robertson, de Pauw, Raynal, Montesquieu oder Rousseau waren gleichsam ,natürliche‘, aber keineswegs ausschließliche Dialogpartner des Jesuiten. Ihre Schriften wurden nicht nur mit Vertretern der spanischen und neuspanischen Aufklärung, sondern auch mit indigenen Quellentexten und eigenen Erfahrungen vor Ort konfrontiert, die freilich aus der Distanz des Exils, die Clavijero zu erwähnen nicht vergisst, oftmals nicht erneut überprüft werden konnten. Doch steht zweifelsfrei fest, dass sie wohl in die Konstituierung eines eigenen kulturellen Raumes ebenso in Hinblick auf das Objekt wie auf das Subjekt (und die Subjekte) dieser Geschichtsschreibung Amerikas einbezogen wurden.

So konnte ein Raum entstehen, der in kultureller Hinsicht weitaus komplexer war als die ausschließlich durch Lektüre erzeugten spekulativen Entwürfe europäischer „Philosophes“, deren politisch-appellative Funktion in den spanischen Kolonien breiten Widerhall fand, deren monokulturell-eurozentrische Konzeption aber bei den kreolischen Eliten nachhaltigen Widerstand hervorrief. Dies war nicht jene universalistisch ausgerichtete Philosophie der Aufklärung, von der die Philosophen Europas gesprochen hatten. Nein, ein kulturphilosophischer Entwurf, der die Amerikaner bestenfalls als Marginalie innerhalb eines evolutiven Prozesses der „Histoire universelle“ verstand, dessen Impulse von Europa ausgingen und nach Europa zurückkehrten, konnte ihren Ansprüchen und Bedürfnissen nach einer Legitimierung jener Protagonisten-Rolle, die zu spielen sie sich in eben jenen Jahrzehnten anschickten, keinesfalls gerecht werden. Die vorgeblich universalistische Perspektive, aus der die Mehrzahl der

6 Vgl. hierzu auch Buche, Irina: Mexikos Dialektik der Aufklärung in den Diskursen von Fray Francisco Javier Clavijero und Fray Servando Teresa de Mier y Guerra. In: Schönberger, Axel / Zimmermann, Klaus (Hg.): De Orbis Hispani linguis et litteris historia moribus. Festschrift für Dietrich Briesemeister zum 60. Geburtstag. Bd. 2. Frankfurt am Main: Domus Editoria Europaea 1997, S. 1300. 
europäischen Philosophen schrieb, war aus amerikanischem Blickwinkel nichts anderes als eine europäische, ja eurozentrische Perspektivik, die sich universalistisch gerierte.

Die auf Amerika gerichtete Differenz, die von den französischen Philosophen des 18. Jahrhunderts fast ausschließlich negativ konnotiert und als Degenerierung gedeutet worden war, sollte nicht etwa verschwinden, sondern positiv umgedeutet werden. Dies bedeutete, dass die ,Abweichung“ vom europäischen Modell nicht als „écart“, sondern in ihrem eigenen Recht als eigener Weg wahrgenommen werden sollte, den die amerikanischen Zivilisationen eingeschlagen hatten. Es gab nicht ein einziges Modell, eine einzige Norm, selbstverständlich diejenigen Europas, die für alle Wege der Zivilisation verbindlich sein mussten, sondern viele Wege, die sich voneinander unterschieden, aber jeweils ihr eigenes Recht beanspruchen durften. Für ein solches Denken aber eignete sich die Rede von einer eigenen, nicht mehr neuspanischen, sondern alt-mexikanischen Antike vorzüglich. Diese Funktionalisierung des Indigenen durch den kreolischen Gelehrten dürfen wir bei aller Bewunderung für die umfangreichen, ja epochemachenden Studien und Überlegungen Clavijeros nicht vergessen; handelte es sich doch um eine „historia polémica, una respuesta a la mirada de los europeos“", ${ }^{7}$ um eine durchaus auch polemische Antwort auf den Blick der Europäer.

Die Konstruktion eines grundsätzlich anderen, vor allem aber weiteren ${ }^{8}$ kulturellen Raumes wird von Beginn der Historia antigua de México an sehr bewusst nicht nur in den Paratexten, sondern auch im Hauptteil dieser „Geschichte des alten (oder vielleicht besser noch des antiken) Mexiko“ betrieben. Denn der kulturelle Raum der Amerikas ist auf Grund der europäischen Eroberung und Besiedelung kein fundamental anderer, da er als ersten kulturellen Pol die okzidentalen Traditionslinien berücksichtigt, aber sehr wohl ein gegenüber dem europäischen deutlich weiterer und erweiterter, weil in ihm kulturelle Pole vorhanden sind, die in Europa fehlen. Sehen wir von weiteren paratextuellen Elementen wie Illustrationen und der Beigabe einer Karte einmal ab, die der Leserschaft das „Anáhuac o Imperio Mexicano con los Reinos de Acolhuacán y de Michuacán \&c.“ zeigt, dann schlägt sich dies bereits im ersten Satz des ersten Kapitels der Historia antigua de México nieder: „Der Name Anáhuac, der gemäß seiner Etymologie anfangs allein auf das Hochtal von Mexiko bezogen

7 Gallardo Cabrera, Salvador: La disputa por la diferencia: acerca de Clavijero, Buffon y la historia natural. In: Cuadernos Americanos (México) XI, 61 (enero - febrero 1997), S. 153.

8 Vgl. zur Kategorie des Weiteren Ette, Ottmar: Weiter denken. Viellogisches denken / viellogisches Denken und die Wege zu einer Epistemologie der Erweiterung. In: Romanistische Zeitschrift für Literaturgeschichte / Cahiers d'Histoire des Littératures Romanes (Heidelberg) XL, 1-4 (2016), S. 331-355. 
war, insofern dessen Hauptansiedelungen an dessen beiden Seiten liegen, wurde später ausgeweitet auf den gesamten Landraum, der heute unter dem Namen Neuspanien bekannt ist.“9 In diesem Eingangssatz spannt Clavijero zunächst jenen geographischen Raum auf, in welchem er die historischen und kulturellen Ereignisse und Phänomene situieren wird, mit denen er sich in seiner Historia antigua de México auseinandersetzt. Er buchstabiert mit seinen Leserinnen und Lesern die verschiedenen Benennungen dieses „espacio de tierra“ durch, um unter der kolonialspanischen Bezeichnung „Nueva España“ gleichsam archäologisch die historischen Tiefenschichten freizulegen, welche durch die gesamte neuspanische Geschichte hindurchwirken.

Auf diese Weise entsteht ein Raum, der einerseits - wie es die Verschiedenartigkeit seiner Benennungen schon andeutet - heterogen ist, der sich aber andererseits als historisch-politische Einheit erhalten hat. Und man könnte hinzufügen: bis zum heutigen Tag. Innerhalb dieser quer zur kulturellen Heterogenität verlaufenden politischen Kontinuität wiederum zeigt sich ein expansiver Prozess, der von den Ufern zweier Seen, vom Hochtal von Mexiko ausgeht und von diesem Zentrum aus eine Einheit schafft, welche sich auf allen Ebenen - von der politischen bis hin zur gastronomischen - weiterzuentwickeln vermochte.

Der Akzent oder das Gewicht dieses ersten Satzes liegt mithin weniger auf der Heterogenität als auf einer Kontinuität, die in grundlegender Weise auf eine Differenz gründet, welche es näher zu erkunden gilt. Die Bezeichnung „Neuspanien“ legt sich nur über die zuvor bereits gegebene Einheit, die das Ergebnis eines historischen Prozesses der Expansion einer bestimmten indigenen Gruppe ist, der Mexica. Damit wird ganz bewusst und absichtsvoll eben jene Bruchlinie unterlaufen, die die bisherige europäische Geschichtsschreibung mit der Conquista ansetzte und die alles zuvor Dagewesene aus dem Bereich der Zivilisation, ja aus dem Bereich der Geschichte verbannt. Der geschichtliche Ausgangspunkt dieses Raumes wird um Jahrhunderte zurückverlegt in die präcortesianische Zeit, die zum Orientierungspunkt für eine aktuelle Geschichte wird, die zunehmend als transitorisch und ephemer, vor allem aber als vom Menschen gestaltbar erfahren werden kann. Denn die Geschichte der amerikanischen Antike hat notwendigerweise Rückwirkungen auf diejenige einer amerikanischen Zukunft.

What's in a name? Die Bezeichnung „Neuspanien“ erscheint als Usurpation, ist dem bereits bestehenden Raum nur übergestülpt, nicht aber aus ihm heraus gewachsen. „Neuspanien“ wird damit zu einer Bezeichnung, die historisch auch

9 Clavijero, Francisco Javier: Historia antigua de México, S. 1: „El nombre de Anáhuac que según su etimología se dio al principio a sólo el valle de México, por estar situadas sus principales poblaciones en la ribera de dos lagos, se extendió después a casi todo el espacio de tierra que hoy es conocida con el nombre de Nueva España.“ 
wieder verschwinden kann und die in der Tat auch wieder verschwand. Ergebnis dieser folgenschweren Umgewichtung ist nicht Heterogenität, sondern kulturelle Differenz und eine damit verbundene Legitimation kultureller und letztlich auch politischer Selbständigkeit, die in der Tat die letztgenannte Bezeichnung (,hoy es conocida con el nombre de Nueva España“) abstoßen und verdrängen wird. Es scheint mir daher verkürzt und der kulturell wie symbolisch komplexen Situation der Kreolen unangemessen, den Rückgriff auf die indianische Geschichte als reine Propaganda (die es sicherlich auch gab und die zweifellos in den Augen der Trägerschicht der Unabhängigkeitsrevolution auch sinnvoll war) zu deuten und damit als etwas nur nachträglich Aufgestülptes zu werten. ${ }^{10}$ Die gesamte Anlage der Historia antigua de México widerspricht einer derart einseitig politischen Zielsetzung und so hat Clavijero für sein Buch auch nicht diese kolonialspanische, sondern die autochthone Bezeichnung „México“ gewählt. ${ }^{11}$

Damit ist die gewollte Aussparung der amerikanischen Kulturen als Kulturen, wie wir sie bei Guillaume-Thomas Raynal, aber auch bei vielen anderen europäischen Autoren des 18. Jahrhunderts konstatieren können, zugunsten einer Konzeption überwunden, die das spezifisch Amerikanische zum Element der Gründung und Begründung eines Eigenen werden lässt. Dabei wird das Europäische - wie etwa die christliche Religion oder die wissenschaftlichen Schreibgattungen - nicht aus diesem Panorama herausgetrennt, was für den jungen neuspanischen Jesuiten und Kulturhistoriker freilich eine Selbstverständlichkeit war. Analog lässt sich dies auch auf den literarischen Raum übertragen, der nicht so sehr als Raum des Heterogenen als vielmehr der Differenz - und zwar einer Differenz im Sinne einer Erweiterung - in Szene gesetzt wird. Dies sichert auch auf dieser Ebene eine Einheit, die zusammen mit der Differenz überhaupt erst die konstruktive Grundlage für eine eigene (tendenziell nationale) Identitätsstiftung schafft.

Die Zentralstellung des Valle de México, die wir bereits im ersten Satz der Historia bemerken konnten, findet sich auf verschiedenen Ebenen des kulturhistorischen und historiographischen Textes wieder. So heißt es etwa im zweiten Kapitel des ersten Buches der Historia antigua de México: „Der beste Teil

10 Vgl. König, Christoph: Staaten - keine Nationen. Die Heterogenität der Kulturen Lateinamerikas. In: Agora (Eichstätt) 2 (1997), S. 8: „Doch läßt die Art und Weise, wie die Kreolen sich selbst in die dreihundert Jahre dauernde Unterdrückung der Indios einbezogen und eine ,gemeinsame‘ Geschichte als Eroberte und Unterdrückte konstruierten, deutlich erkennen, daß die Kreolen die Existenz der Indios lediglich zu Propagandazwecken nutzten, um die Überwindung von Unfreiheit als Ziel der Bewegung angeben zu können.“

11 Häufig findet sich in seiner Historia nach der Einführung einer indigenen Bezeichnung der Hinweis auf den spanischen Ortsnamen, der zumeist mit der Formel „que los españoles dicen ...“ eingeleitet wird. 
dieses Landes - und zwar ebenso durch seine vorteilhafte Lage wie durch seine größeren Bevölkerungsballungen - war eben das Hochtal von Mexiko, welches rundherum von großen grünen und herrlichen Bergen gekrönt wird. ${ }^{12}$

Diese Orientierung am Hochtal, am Valle de México hat von Beginn an kulturelle wie politische Implikationen, werden doch schon auf den ersten beiden Seiten andere indigene Völkerschaften als „barbarisch“ bezeichnet. So ist etwa von den „bárbaros chichimecas“13 oder von einem vom Hochtal entfernten Gebiet die Rede, das „estaba ocupado de bárbaros que ni tenían domicilio alguno ni reconocían soberanos“, ${ }^{14}$ das also nicht sesshaft und keinem Souverän untergeordnet war.

Nach diesen zunächst überraschenden Worten wird auf Ebene der amerikanischen Völkerschaften eine kulturelle Hierarchie eingeführt, deren Unterscheidung zwischen ,zivilisierten“ und ,barbarischen“ Stämmen an Kriterien ausgerichtet bleibt, die - wie etwa Sesshaftigkeit, feste politische Struktur, Schriftlichkeit oder Geschichtsschreibung - von den europäischen Autoren des 18. Jahrhunderts zwar nicht erfunden, wohl aber mit dem Neologismus „civilisation“ fest verbunden wurden. Dies mag die Richtigkeit der Bemerkung Salvador Gallardos belegen, der darauf aufmerksam machte, dass sich nicht nur die europäischen, sondern alle an der Disputa um die Deutung der Neuen Welt beteiligten Autoren auf dieselbe und gemeinsam geteilte Vernunft beriefen. Auch ein Clavijero war noch weit entfernt von der Einsicht, dass dieser abendländischen Vernunft totalitäre Züge zukommen könnten ${ }^{15}$ - zumindest im Sinne von Max Horkheimers und Theodor W. Adornos Dialektik der Aufklärung.

Kritisierte Francisco Javier Clavijero auch bitter die Haltung der Europäer, alle amerikanischen Völker über denselben Leisten zu schlagen - „los críticos de Europa, acostumbrados a medir por un rasero a todas las naciones americanas“16 -, so nutzte er die dadurch ermöglichte Differenzierung doch nur wieder, um innerhalb der amerikanischen Völker Hierarchien (etwa zwischen „cultos“ und „bárbaros“) zu errichten. Andererseits unterlag er immer wieder der Versuchung, eine von ihm konstatierte Heterogenität im Kontrast zu den Spaniern zumindest tendenziell in Homogenität umzudeuten.

12 Clavijero, Francisco Javier: Historia antigua de México, S. 2: „La porción mejor de esta tierra, así por su ventajosa situación como por sus grandes poblaciones, era el mismo valle de México, coronado por todas partes de verdes y hermosas montañas.“

13 Ebda.

14 Ebda., S. 1.

15 Gallardo Cabrera, Salvador: La disputa por la diferencia, S. 155.

16 Clavijero, Francisco Javier: Historia antigua de México, S. 50. 
Charakteristisch hierfür ist der Beginn des 17. Kapitels seiner Naturgeschichte des Landes Anáhuac, die das erste Buch seiner Historia bildet:

\begin{abstract}
Jene Nationen, welche diese Landstriche vor den Spaniern besaßen, waren zwar untereinander in ihrer Sprache und teils auch in ihren Sitten sehr voneinander unterschieden, doch waren sie gleichsam von ein und demselben Charakter. Die physische und moralische Konstitution der Mexikaner, ihre Einbildungskraft wie ihre Neigungen, waren dieselben bei den Acolhuas, den Tlaxkalteken, den Tepaneken und den anderen Nationen, ohne einen anderen Unterschied als den, welche eine verschiedenartige Erziehung hervorbringt. Und was ich so von den einen sage, möchte ich so auch bezüglich der übrigen verstanden wissen. Verschiedene Autoren der alten wie der modernen Zeit haben es unternommen, ein Portrait dieser Nationen anzufertigen; doch unter so vielen hat sich nicht eines gefunden, das präzise und in allen Aspekten getreu wäre. Leidenschaft und Vorurteile bei den einen Autoren sowie der Mangel an Kenntnissen und an Reflexion bei anderen haben sie Farben verwenden lassen, welche sich von denen sehr unterschieden, die sie hätten verwenden müssen. Was ich sagen werde, basiert auf einem ernsthaften und umfangreichen Studium ihrer Geschichte sowie auf einem intimen und jahrelangen Umgang mit den Mexikanern. Andererseits entdecke ich in mir nichts, was mich für oder gegen sie hätte einnehmen können. Weder die Vernunft eines Landsmannes neigt mein Unterscheidungsvermögen zu ihren Gunsten, noch führen mich die Liebe zu meiner Nation oder der Eifer, den Angehörigen meiner Nation die Ehre zu erweisen, zu ihrer Verurteilung; und so werde ich frei und Ehrlich das Gute wie das Schlechte sagen, das ich an ihnen erkannte. ${ }^{17}$
\end{abstract}

Die Formulierung mit dem Verweis auf die „antiguos“ wie die „modernos“ kommt nicht von ungefähr: Clavijero war bezüglich der Auseinandersetzungen im Frankreich der Frühaufklärung (oder auf dem Weg zur Frühaufklärung) durchaus auf dem Laufenden. Überhaupt teilte er mit den Autoren der europäischen Aufklärung wesentliche Grundüberzeugungen. Es wäre ein Leichtes, an dieser Passage die Übereinstimmung mit grundlegenden, von der europäischen Aufklärung propagierten Werten wie Unabhängigkeit, Wahrheitsanspruch, Zerstörung von Vorurteilen, wissensbasierte Kritik oder objektive Interesselosigkeit nachzuweisen, die hier aber nun gerade gegen die Europäer und die europäische Aufklärung ins Feld geführt werden. Wie die haitianischen schwarzen Sklaven die Argumente der Französischen Revolution gegen ihre gleichwohl fortgesetzte Versklavung und damit gegen die europäischen Revolutionäre in Stellung brachten, so ging es auch Clavijero darum, die von der europäischen Aufklärung propagierten Werte auch auf die europäische Aufklärung selbst zu beziehen und anzuwenden.

Genau an dieser Stelle befindet sich der eigentliche Kern der Aussagen des neuspanischen Jesuiten: Hier liegt sozusagen der ,philosophische Hund' begra-

17 Ebda., S. $44 \mathrm{f}$. 
ben. Denn die neuspanische - wie die hispanoamerikanische - „Ilustración“ bedient sich der Vorstellungen und Grundwerte der europäischen Aufklärung, partizipiert daran und muss sie doch im Sinne ihres eigenen Standpunktes im Grunde noch extremer verwirklichen, noch radikaler einsetzen, wobei sie ihre eigene ideologische und ideengeschichtliche Basis gefährdet. Doch ging es darum, das von den europäischen Aufklärern ganz selbstverständlich beanspruchte Recht, von Europa aus alle Erscheinungen auf dem Planeten beurteilen zu können, einer Kritik zu unterziehen und damit einen europäischen ,Universalismus', der sich zumindest selbst als universalistisch ausgab, grundlegend zu hinterfragen.

In der obigen Passage erscheint zugleich die Idee der menschlichen Formbarkeit durch Erziehung, die - kaum überraschend für einen Jesuiten - ,vervollkommenbare' Gestaltung des menschlichen Geistes durch Bildung und Ausbildung am Horizont eines evolutionistischen Denkens, das Heterogenität nicht als Chance, sondern eher als Gefahr für die Einheit versteht. Die Historia antigua de México kann ohne den Kontext der polemischen Auseinandersetzung mit Raynal, Robertson und de Pauw - wobei letzterer als bevorzugte (da am leichtesten zu treffende) Zielscheibe die schärfste Kritik auf sich zog - nicht adäquat verstanden werden. Doch dient der Rückgriff des kreolischen Jesuiten auf die ,eigene', die amerikanische Antike gerade nicht der Ausgestaltung einer Hybridität von Kulturen amerikanischer und europäischer Provenienz. Wir werden uns noch wiederholt im weiteren Verlauf der Vorlesung mit den Schriften des Cornelius de Pauw zu beschäftigen haben, doch sei in dieser Vorlesung zunächst einmal die amerikanische Seite gehört. Ich bitte Sie also noch um eine Weile Geduld, bevor wir uns den Thesen des in Amsterdam geborenen Denkers gleich auf den nächsten Seiten zuwenden!

Clavijeros letztlich erfolgreich und überzeugend geführter Kampf gegen die Ausgrenzung, Verdrängung und Leugnung der amerikanischen Hochkulturen durch europäische Philosophen wie etwa Guillaume-Thomas Raynal - die er polemisch als „la franqueza de un filósofo del siglo XVIII“18 brandmarkt - verfolgt ohne jeden Zweifel das Ziel, eine kulturelle Alterität und politische Legitimität der Bewohner Neuspaniens und insbesondere der Kreolen zu begründen. Dies beinhaltete nicht die kulturelle und politische Einbeziehung der präsenten (und nicht historischen) indigenen Völker, sondern macht eine Differenzposition gegenüber Europa auf, deren Ratio, deren Vernunft sich Clavijero als Bewohner der Ciudad letrada in Amerika gleichwohl verpflichtet weiß. So wird jenseits aller Polemik ein Europäern und Kreolen gemeinsamer diskursiver (und kultureller) Raum nicht verlassen: Clavijero versteht und weiß sich als

18 Ebda., S. xxxiii. 
Mitglied jener Gemeinschaft aufklärerischer Geister, die sich zwischen Europa und den Amerikas zu etablieren begann.

Seine Überlegungen müssen im Kontext jener zweiten Phase beschleunigter Globalisierung, die mittlerweile längst eingesetzt hatte, und jener Auseinandersetzungen verstanden werden, die vom Selbstverständnis eines in ständiger Expansion begriffenen Europa ausgelöst wurden, das unter seinen verschiedenen Kolonialmächten die Welt neu aufzuteilen begonnen hatte. Es mag vielleicht kein Zufall sein, dass mit dem Franzosen Raynal, mit dem Schotten Robertson und mit dem Holländer de Pauw Philosophen aus jenen Nationen an der Disputa del Nuovo Mondo beteiligt waren, die ihre jeweiligen Kolonialreiche längst auszuweiten begonnen hatten und zugleich in ständigen Konflikten untereinander standen. Längst hatten sie - wie etwa die Kolonialmacht Frankreich in Saint-Domingue, dem späteren Haiti - ihre Ausbeutungssysteme weiter modernisiert und neue Formen und Varianten des europäischen Kolonialismus erprobt.

Die andere, philosophische und ideengeschichtliche Seite dieser Expansion ist der Universalismus, die universalisierende Tendenz eines Denkens, das aus der Erfahrung der Überlegenheit europäischer Konzeptionen zugleich auch das unheimliche (im Sinne Freuds) und ungeheure Recht ableiten zu können glaubt, über die allein seligmachenden, allein das wahre Menschsein entwickelnden Vorstellungen zu verfügen. Die eigene Entwicklung wird mit der eigentlichen Hauptentwicklungsachse des Menschengeschlechts schlechthin gleichgesetzt. Entscheidend für ein solches Denken ist im Sinne Tzvetan Todorovs, ${ }^{19}$ dass es im Grunde implizit sofort den Gedanken der Überlegenheit und der Unterlegenheit mitzutransportieren beginnt, wobei in diesem Falle die Inferiorität nach Amerika projiziert wurde.

Es handelte sich dabei um die Vorstellung einer grundlegenden Unterlegenheit der Amerikaner, die übrigens mit dem Ende des 18. Jahrhunderts als europäische Projektion noch keineswegs verschwand. Dies gilt keineswegs nur für so zentrale Philosophen wie Georg Friedrich Wilhelm Hegel, sondern auch hinsichtlich eines für den Marxismus so essentiellen Denkers wie Friedrich Engels. Er billigte etwa im Zuge seiner Aufwertung des Nordens den noch jungen, aber höchst dynamischen Vereinigten Staaten von Amerika das Recht zu, sich nach Westen und Süden auszudehnen, um so Völker einer unterlegenen Kultur in den Entwicklungsgang der Weltgeschichte mithineinzunehmen oder, besser vielleicht noch, in diese von den Völkern des Nordens gelenkte Strömung der Weltgeschichte hineinzureißen.

19 Vgl. hierzu Todorov, Tzvetan: La conquête de l'Amérique. La question de l'autre. Paris: Seuil 1982. 
Nach der Abwertung der amerikanischen Natur in toto durch den Franzosen Buffon, den Verfasser der berühmten Histoire naturelle, war sicherlich der in Amsterdam geborene Cornelius de Pauw mit seinen Recherches philosophiques sur les Américains der entscheidende Autor für eine Abwertung alles ursprünglich Amerikanischen im Bereich des Menschen, also innerhalb einer universalen Anthropologie des europäischen 18. Jahrhunderts. Der erste Band dieses Werks, mit dem wir uns noch gesondert auseinandersetzen werden, ist auf Berlin im Jahre 1768 datiert. De Pauw stand ganz in der Linie der französischen Enzyklopädisten - nicht nur auf Grund seiner ständigen Angriffe gegen die Religion und speziell gegen die Jesuiten, deren Vertreibung aus Spanien und aus den spanischen Kolonien zeitgenössisch gerade abgeschlossen beziehungsweise noch im Gange war. Denn Cornelius de Pauw vertrat auch in einer absoluten Form das Vertrauen auf den materiellen wie geistigen Fortschritt der Menschheit sowie eine grundlegende Skepsis gegenüber jedweder behaupteten Existenz einer natürlichen Güte des Menschen. Die notwendigen Konsequenzen dieser Position gerade auch für den europäisch-amerikanischen Dialog - wenn von einem solchen in einem gleichberechtigten Sinne denn gesprochen werden kann - sind leicht auszumalen, da er der Inferioritätsthese alles Amerikanischen im Gefolge Buffons breitesten Raum gab.

Die philosophische Positionen des Holländers de Pauw, der übrigens von 1739 bis 1799 lebte und damit die Französische Revolution - gleichsam in der dritten Generation der „Philosophes“ - noch erleben konnte, ist in vielerlei Hinsicht bedeutsam geworden. Sein Anti-Rousseauismus war unverkennbar: Er hielt nichts von einem Rückzug des Menschen in die Natur, schrieb dieser auch keinerlei positiven Einfluss auf den Menschen zu, erwartete sich aber alles von der Entwicklung der Zivilisation und vom Einfluss der Gesellschaft auf den einzelnen Menschen. ${ }^{20}$ Ohne seine Kultur, ohne eine ständige Kulturarbeit, die an ihm vollzogen werde, war der Mensch im Sinne de Pauws, der seine Vorstellungen auch von Potsdam und Berlin aus in der Berliner Debatte um die Neue Welt vertrat, schlicht nichts.

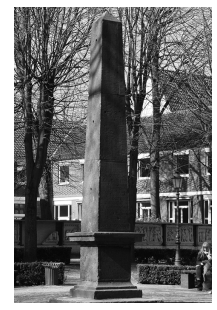

Abb. 29: Obelisk zu Ehren Cornelius de Pauws in Xanten.

20 Vgl. hierzu Gerbi, Antonello: La Disputa del Nuovo Mondo, S. 71. 
Schon für den Grafen Buffon war die Natur der amerikanischen Hemisphäre, zumindest in ihrer tropischen Variante, eine ihrem Alter nach jüngere, ,neue' Natur, die sich noch nicht hatte stark entwickeln können und zugleich degeneriert war. Der Begriff der „Neuen Welt“ wurde auch geologisch verstanden in einem Sinne, demzufolge diese Welt neueren Datums wäre und sich noch nicht vollständig aus den vielen Flussgebieten, Seen und Sümpfen heraus habe entwickeln können. Die Übertragung einer solchen Anschauung vom natürlichen auf den menschlichen Bereich, also von Flora und Fauna auf den Menschen lag nahe. Diese naturhistorische (und damit dem damaligen Stand der europäischen Wissenschaft entsprechende) Übertragung erfasste in der Folge alle kulturellen und ethnischen Gruppen der Neuen Welt, die indigenen Völkerschaften ebenso wie die Kreolen, die im Übrigen natürlich auch wegen des tropischen Klimas der Degenerierung anheimfallen würden. Alle Bereiche der Natur wie der Kultur - und damit alle Bereiche des menschlichen Lebens wurden vom Klima der Tropen geprägt: So glaubte man, selbst in der Literatur einen vom tropischen Klima geprägten Stil erkennen zu können. ${ }^{21}$

Nur nebenbei sei bemerkt, dass auch Montesquieu zu den Hauptvertretern einer Klimatheorie zählte, die von der ersten Hälfte des 18. Jahrhunderts bis tief ins 20. Jahrhundert und wohl auch bis tief ins kollektive Unbewusste der Europäer des 21. Jahrhunderts fortwirken konnte und weiter fortwirkt. Ganz so fern sind uns die Polemiken des 18. Jahrhunderts bei weitem nicht. Für Cornelius de Pauw jedenfalls stehen Amerika und Europa sich als zwei Teile ein und desselben Globus gegenüber, die im Grunde wenig miteinander zu tun haben, da sie repräsentativ für gegenteilige Phänomene innerhalb dieser unserer einen Welt stehen. ${ }^{22}$ De Pauws Vorstellungen gingen mithin deutlich über eine Replik auf die von ihm kritisierten Berichte der Missionare und über eine Grundsatzkritik an der Vorstellung vom „bon sauvage“ hinaus. Die Degenerierung alles Amerikanischen manifestierte sich für de Pauw auf allen Bereichen, ebenso in der körperlichen und physischen Schwäche der amerikanischen Bevölkerungen wie in der Unterlegenheit an Intelligenz und kognitiven Fähigkeiten einer

21 Das Fortdauern derartiger Konzepte des 18. Jahrhunderts im nachfolgenden 19. Jahrhundert hat aufgezeigt Ventura, Roberto: Estilo tropical. História cultural e Polêmicas literárias no Brasil 1870 - 1914. São Paulo: Companhia das Letras 1991.

22 Vgl. hierzu auch Gerbi, Antonello: La Disputa del Nuovo Mondo, S. 79; sowie allgemein zu de Pauw Ette, Ottmar: Wörter - Mächte - Stämme. Cornelius de Pauw und der Disput um eine neue Welt. In: Messling, Markus / Ette, Ottmar (Hg.): Wort Macht Stamm. Rassismus und Determinismus in der Philologie (18. / 19. Jh.). Unter Mitarbeit von Philipp Krämer und Markus A. Lenz. München: Wilhelm Fink Verlag 2013, S. 107-135. 
,Rasse‘, welche sich vollständig und unausweichlich in einer Phase völliger Dekadenz befinde. Gerade mit Blick auf seine folgenschweren Äußerungen zu verschiedenen ,Rassen“ in seinem Bestseller Recherches philosophiques sur les Américains übte Cornelius de Pauw weit über das Aufklärungszeitalter hinaus einen langanhaltenden Einfluss auf ganz Europa aus.

Seine Ansichten führten gleich nach der Publikation der Recherches in Berlin im Jahr 1768 zu vielfältigen Polemiken in Europa, insbesondere mit Pernety, auf den de Pauw freilich in seiner ebenfalls auf Berlin im März 1770 datierten Défense des Recherches philosophiques sur les Américains antwortete. Ich werde in dieser Vorlesung noch detailliert auf die Berliner Debatte um die Neue Welt eingehen. Doch so viel vorab: De Pauw wertete ganz so, wie dies auch Buffon oder Diderot getan hätten, die bloße Beobachtung der sogenannten „faits“, der Tatsachen also ab; hinderten diese doch nur daran, die wahren Ursachen und Entwicklungen zu erkennen. ${ }^{23}$ Man war noch meilenweit entfernt von einer empirischen Fundierung der Wissenschaften und von durch Feldforschung erzielten Ergebnissen, wie sie dann zunehmend an der Wende zum 19. Jahrhundert in den Vordergrund treten sollten.

Im Laufe der Polemik modifizierte de Pauw seine ursprüngliche These von der generellen Degenerierung der Amerikanischen Völker dahingehend, dass er nun behauptete, die Indianer seien zum Zeitpunkt der Eroberung durch die iberischen Mächte in voller Dekadenz gewesen; nun aber - seit der Eroberung durch die Spanier - habe eine neue Entwicklung begonnen, die wiederum im Zeichen eines beginnenden Fortschritts stehe. Aus diesem Grunde seien die vergangenen dreihundert Jahre also keineswegs verlorene Jahre, denn die Europäer hätten die amerikanischen Völker wieder auf den Weg des Fortschritts gebracht - und dies sei der vorgezeichnete Weg der Menschheit überhaupt. Dazu merkte der holländische, in preußischen Diensten stehende Kleriker an: „Nach dreihundert Jahren wird Amerika nur noch wenig mit dem übereinstimmen, was es heute ist, so wie es heute nur noch wenig mit dem übereinstimmt, was der Kontinent zum Zeitpunkt seiner Entdeckung war.“24

Mit diesen Überlegungen wurde der Entwicklungsgedanke auch den Amerikanern, also vorrangig der indigenen Bevölkerung Amerikas zugebilligt, was durchaus einen grundlegenden Unterschied zu den ersten Stellungnahmen und

23 Vgl. hierzu auch Gerbi, Antonello: La Disputa del Nuovo Mondo, S. 132.

24 De Pauw, Cornelius: Défense des Recherches philosophiques sur les Américains, ou Mémoires intéressants pour servir à l'Histoire de l'Espece humaine. Berlin: Chez J. G. Baerstecher 1772, Bd. 2, S. 112: „Au bout de trois-cents ans, l'Amérique ressemblera aussi peu à ce qu'elle est aujourd'hui, qu'elle ressemble aujourd'hui peu à ce qu'elle étoit au temps de la découverte.“ 
Einschätzungen de Pauws darstellte. Doch seine grundlegenden Thesen, Behauptungen und Überzeugungen änderten sich keineswegs, wie auch der weitere Verlauf der internationalen Diskussion, der „Disputa“ zeigen sollte. Antonello Gerbi wies mit Recht darauf hin, dass sich Voltaire in einer ersten Reaktion für die Thesen von de Pauw erwärmen konnte und den Holländer als einen „vrai philosophe“, als wahren Philosophen bezeichnete. ${ }^{25}$ Doch de Pauw ließ auch auf anderen Gebieten nicht locker und veröffentlichte fünf Jahre nach seinen Recherches philosophiques über die Amerikaner nun ebensolche über die Ägypter und Chinesen: Er war längst zu einer europäischen Berühmtheit geworden.

Charakteristisch für die Rezeption der Recherches war eine Rezension von Jacobi, in welcher der Rezensent die Talente und den Stil des Holländers lobte. ${ }^{26}$ Auch stellte er manche Übereinstimmungen mit den Überzeugungen Winckelmanns fest. Jacobi enthielt sich explizit und willentlich aller tiefer gehenden Kritik. Es erstaunt zudem in keiner Weise, dass auch Immanuel Kant einem Briefpartner die erweiterte Ausgabe der Recherches philosophiques sur les Américains empfahl. ${ }^{27}$ Herder und Kant nahmen de Pauws Überlegungen zu den Ägyptern voller Respekt auf. Der holländische Abt hatte mit seinen polemisch ausgerichteten, scharfzüngigen Schriften nicht nur einen kurzfristigen, sondern einen langfristig sich auswirkenden nachhaltigen Erfolg, dessen Einfluss auf die Entfaltung des Rassedenkens wie des Rassismus keineswegs nur in Deutschland, sondern in ganz Europa von fundamentaler Bedeutung war.

Freilich gab es auch in Europa einige Gegenstimmen: Voltaire etwa distanzierte sich zunehmend ebenso von den Thesen de Pauws gegenüber den Amerikanern, also der indigenen Bevölkerung der Amerikas, wie von jenen gegenüber Ägyptern und Chinesen. Voltaire tat dies nicht nur, weil de Pauw ohne jede Kenntnis vor Ort behauptete, die Chinesen seien von den Ägyptern kolonisiert worden. Immerhin war De Pauw von keinem geringeren als dem Preußenkönig Friedrich dem Großen protegiert worden; und dieser berichtete 1776 amüsiert an Voltaire, de Pauw sei ganz eitel und stolz darauf, dass Voltaire ihm einige Briefe gewidmet habe. ${ }^{28}$ Wir werden uns im Anschluss noch etwas näher mit den Beziehungen zwischen Cornelius de Pauw und Friedrich dem Großen beschäftigen, freilich weniger auf einer persönlichen als auf einer strukturellen Ebene.

Zwar ist Cornelius de Pauw heute längst vergessen und nur noch wenigen Spezialisten für das 18. Jahrhundert bekannt. Doch er hat das europäische Denken über die außereuropäische Welt entscheidend mitgeprägt und nicht nur

25 Vgl. Gerbi, Antonello: La Disputa del Nuovo Mondo, S. 211.

26 Vgl. ebda., S. 213.

27 Vgl. ebda.

28 Vgl. ebda., S. $216 \mathrm{f}$. 
kurzfristig die entstehende öffentliche Meinung beeinflusst, sondern langfristig gerade im Bereich der Philosophie gewirkt. Nicht nur ein Kant, sondern vor allem ein Hegel nahmen seine Thesen begierig auf - sehr zum Ärger Alexander von Humboldts, der über Hegels Rede von den degenerierten und schwächlichen Krokodilen in Amerika herrlich spottete. Es stünde der Philosophiegeschichte in Deutschland gut zu Gesicht, wenn sie nicht allein die Frage von Immanuel Kants Rassismus, die noch immer in den Medien heiß diskutiert wird, sorgfältig wissenschaftlich klärte, sondern auch diese intensive Beziehung zwischen Hegels weltgeschichtlicher Sichtweise und den Thesen de Pauws stärker - und unter Einbeziehung der amerikanischen Aufklärung - aufarbeiten würde. Humboldt, der zunächst mit hohen Erwartungen Hegels Vorlesungen an der noch jungen Berliner Universität hörte, hatte jedenfalls ohne weiteres erkannt, woher der große deutsche Philosoph seine Einsichten über die so degenerierten Krokodile und Alligatoren Amerikas bezogen hatte und vor welchem Hintergrund der globale Denker der Weltgeschichte die indigenen Völker Amerikas aburteilte.

Lassen Sie mich an dieser Stelle unsere kurze erste Beschäftigung mit Cornelius de Pauw abschließen und zwei knappe Passagen aus zwei unterschiedlichen Ausgaben der Recherches philosophiques sur les Américains zitieren, welche einerseits seine Abwertung des Naturzustandes, den Rousseau ja mitpropagiert hatte, und zugleich auch seine Begründung für die militärische Durchsetzung der Europäer und des Genozids an den Indianern zeigen:

Alle Gewalt \& alle Ungerechtigkeit waren auf Seiten der Europäer: Die Amerikaner hatten nur ihre Schwäche, so mussten sie ausgelöscht werden, \& wurden ausgelöscht in einem Augenblick. [...]

Er ist in der Welt allein \& weiß nicht, dass man wohltätig, barmherzig \& großzügig sein kann. Man könnte sich keine größere Verrohung unserer Natur vorstellen als diesen Zustand des Nichtstuns \& der Trägheit, in welchem man die Tugend, Ghutes zu tun, nicht kennt \& in dem man sich damit beschäftigt, lediglich an sich oder an seine Herren zu denken. ${ }^{29}$

Der Naturzustand hat für de Pauw also ebenso wenig etwas Anheimelndes wie eine kulturelle Lage, in der die Entwicklung der Menschheit letztlich darauf abzielt, dass sich die überlegene Kraft und Kultur der Europäer gleichsam geschichtsphilosophisch legitimiert und sie - wenn auch unter Selbstanklagen

29 Pauw, Cornelius de: Recherches philosophiques sur les Américains, ou Mémoires intéres-

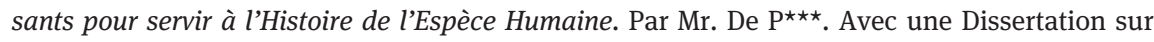
l'Amérique \& les Américains, par Dom Pernety. Bd. 1. London 1775, S. IV f.; sowie (ders.): Recherches philosophiques sur les Américains, ou Mémoires intéressants pour servir à l'Histoire de

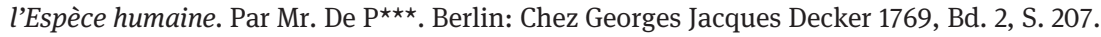


späterer Generationen - auf Kosten aller anderen Kulturen durchsetzt, die in Amerika existieren. Cornelius de Pauw, der im Übrigen als Beiträger für die Encyclopédie sowohl an den Artikeln über die Besiedlung Amerikas wie auch an jenen über China beteiligt war und sich nicht besonders um die damals bekannten Fakten, sondern allein um die Verteidigung seiner Vorstellungen kümmerte, hatte einen enormen Einfluss auf die öffentliche Meinung in ganz Europa. Gegen diesen Einfluss, ja gegen diese Meinungsführerschaft waren zuvor bestenfalls die Stimmen der Jesuiten lautgeworden, die durch Berichte ihrer Ordensbrüder sowohl in Amerika als auch in China über die weitaus besseren Informationen verfügten.

$\mathrm{Zu}$ diesen Stimmen zählte zweifellos auch jene Francisco Javier Clavijeros, zu dem wir nach unserem kurzen ersten Ausflug zu de Pauw wieder zurückkehren dürfen. Clavijero betonte immer wieder seine Augenzeugenschaft, betonte stets sein eigenes Sehen, was ihm eine große Legitimität verschaffte; zumal er von Europa aus schrieb und seine Geschichte, seine Historia antigua de México doch in Italien und auf Italienisch ausarbeitete. Häufige Verweise auf den frühen Altamerikanisten Carlos de Sigüenza y Góngora sowie auf eine Reihe von Sammlungen und „Códices“ belegen zugleich, dass diese Augenzeugenschaft stets durch andere authentische Quellen ergänzt wurde.

Aufschlussreich ist dabei, dass Clavijero immer wieder betonte, dass die Menschen Amerikas („hombres de América“) genauso seien wie jene Europas, nur dass die fehlende Erziehung und andere Zeitumstände der Unterdrückung ihnen nicht erlaubten, die für ihre Entwicklung notwendigen „luces“ zu ihrer Erhellung zu erwerben. ${ }^{30}$ Die von Clavijero aus den verschiedensten Quellen abgeleitete Geschichte ist zunächst eine der Herrscher, vor allem aber auch der von diesen bewerkstelligten Expansionen, wobei diese Geschichte nicht nur narrativiert, sondern auch durch zahlreiche fingierte Ansprachen indigener Sprecher rhetorisch ausgeschmückt wird. Die Frühzeit der Mexica vom See im Hochtal und ihrem Tenochtitlán als beginnendem Machtzentrum wirkt in der Historiographie Clavijeros wie die frühe Phase der Geschichte Roms und der Expansion der Römer über immer größere, umfangreichere Regionen Italiens. Die ganz bewusst eingefügten Parallelen sind offenkundig: Clavijeros Historia antigua de México ist, der Zeit und seinem Status als Jesuit gemäß, eine Herrschergeschichte, die sich aus verschiedenen Quellen speist und die Geschichtsschreibung des Spaniers Torquemada quellenkritisch in Frage stellt. Für lange Zeit - und nicht nur bis zu Alexander von Humboldt, der sie lobte und immer wieder benutzte - sollte sie

30 Clavijero, Francisco Javier: Historia antigua de México, S. 78. 
der Bezugspunkt für die präkolumbische Geschichtsschreibung im Umfeld der entstehenden Altamerikanistik bleiben.

Clavijeros Historia antigua de México liest sich wie eine Geschichte der heroischen Zeit der Römer, wobei gerade die Herauspräparierung bestimmter Heldengestalten im Grunde einer neoklassizistischen Ästhetik folgte, wie sie in der zeitgenössischen kolonialen Literatur an der Tagesordnung war. Nichts deutet auf entstehende präromantische Entwicklungen, wie sie gerade bei der Gestaltung der Heldensagen leicht hätten Anwendung finden können.

Eine besondere Bedeutung kommt dieser Art der Geschichtsschreibung insoweit zu, als sie den Aufstieg der Mexica und ihrer Stadt Tenochtitlán von einer völlig unbedeutenden Ansiedlung, die anderen Völkern Tribut zahlen musste, zur Herrin über den ganzen See von Tenochtitlán und der zentralen Bereiche des Hochtals von Mexiko in fast epischer Breite darstellt. Spannend und bisweilen schillernd sind nicht nur die ,Mexikaner im engsten Sinne, sondern auch manch andere mythische Gestalt, allen voran der König von Texcoco, Nezahualcóyotl, der bis heute als einer der großen Dichter in der lyrischen Tradition Mexikos gilt und dessen Name bis heute nichts von seinem Glanz verloren hat.

Gerne möchte ich Ihnen eine längere Passage aus diesem Abschnitt des vierten Buchs der Historia antigua de México vorführen, um damit das Idealbild eines gleichsam aufgeklärten Herrschers in präkolumbischer Zeit zu evozieren und vorzustellen. Nezahualcóyotl war durch sein engagiertes Eintreten für die mexikanische Sache nach dem Sieg der Azteken über die Tepaneken wieder in seine alten und im zustehenden Rechte als König von Texcoco eingesetzt worden. Und dieses Reich nun organisierte und lenkte er mit dem Gestus eines aufgeklärten Herrschers:

Nezahualcóyotl war neben der einzigartigen Liebe, die er gegenüber seiner Nation empfand, mit einer seltenen Umsicht begabt, und er gab solche Vorsehungen für die Reform des Königreiches, dass man es binnen weniger Jahre viel mehr aufblühen sah als in den Zeiten seiner Vorgänger. Eine neue Form gab er den Räten, welche sein Großvater eingerichtet hatte, und besetzte wichtige Stellen mit Personen, die er als dafür geeignet fand.

Es gab einen Rat für die rein zivilen Angelegenheiten, und unter anderem waren in ihm fünf Herren zugegen, die ihm in der Zeit seiner Schwierigkeiten sehr treu geblieben waren; einen anderen gab es, der für Verbrechensangelegenheiten zuständig war, dem zwei Fürsten vorstanden, die seine Brüder und von hoher Integrität waren. Der Kriegsrat wiederum setzte sich aus den berühmtesten Kapitänen zusammen, unter denen den Vorsitz sein Schwiegersohn hatte, der Herr von Teotihuacán, der General im Heere und einer der dreizehn Helden des Königreiches war. Der Finanzrat bestand aus den Kämmerern des Königs und den hauptsächlichen Händlern am Hofe; drei waren die vorstehenden Kämmerer, zu deren Aufgabe die Eintreibung der Tribute und königlichen Renten zählte.

Er schuf eine Art Akademie der Dichtkunst, der Astronomie, der Musik, der Malerei, der Geschichtsschreibung und der hellseherischen Künste und berief in sie die geschick- 
testen Professoren des Königreiches; diese versammelten sich beizeiten an einem bestimmten Ort, um ihre Erhellungen und Lehrmeinungen auszutauschen, und für jede einzelne dieser Wissenschaften und Künste gründete er Schulen bei Hofe. Was die mechanischen Künste angeht, so teilte er die Stadt Tezcoco in über dreißig Stadtviertel und gab jeder Kunst das ihre, unter Ausschluss der anderen; in einem Viertel waren die Silberschmiede, in einem anderen die Edelsteinschleifer, in wieder einem anderen die Sticker und so die übrigen alle.

Zur Förderung der Religion erbaute er verschiedene Tempel, ernannte Oberpriester für den Kult ihrer Götter, gab ihnen Häuser und teilte ihnen Renten für ihren Unterhalt und für die übrigen religiösen Ausgaben zu; und für den größeren Glanz seines Hofes führte er große Gebäude innerhalb wie außerhalb der Stadt auf und schuf neue Gärten und Forste, die zum Teil viele Jahre nach der Eroberung noch bestanden, und bis heute bemerkt man manche Überreste jener Großartigkeit. Ich bitte die Leser, meinen Glauben an diese und andere ähnliche Gegenstände nicht zu verurteilen, bevor sie nicht das erfahren haben, was wir in dieser Geschichte noch an anderem sagen werden, insbesondere auch in unseren Dissertationen. ${ }^{31}$

In diesem Zitat entwirft Francisco Javier Clavijero das Bild einer präkolumbischen Gesellschaft, die sich nach kriegerischen Auseinandersetzungen wieder sehr gut zu organisieren weiß und von einem Staatsmanne gelenkt wird, der mit den „luces“ seiner Zeit und damit ganz im Sinne der Aufklärung als absoluter Monarch und Wohltäter seines Volkes wirkt und herrscht. Es scheint mir offensichtlich, dass die Bedeutung dieser Passage sowie der Schlussappell an die Leserschaft der Historia antigua de México erst in vollem Maße verständlich und nachvollziehbar wird aus dem Kontrast gegenüber dem im 18. Jahrhundert in Europa vorherrschenden Diskurs über Amerika und die Amerikaner. Denn dieser hatte in der Nachfolge eines Buffon, der selbstverständlich die außereuropäische Welt niemals gesehen hatte, ein Bild der amerikanischen Natur wie der Bewohner Amerikas bei Tieren wie bei Menschen entfaltet, welches von Schwäche, Unvermögen und fehlenden körperlichen wie kognitiven Fähigkeiten gekennzeichnet war. Wie sollte man da einem Jesuiten glauben, der behauptete, in der Zeit vor der Entdeckung Amerikas habe es rational entworfene und ,modernisierte' Staatswesen in den Amerikas gegeben?

Gegen die radikale Abwertung alles Amerikanischen und aller amerikanischen Kulturen aber zog die Historia antigua de México entschlossen zu Felde. Clavijero wusste, dass er angesichts der vorherrschenden Meinung und dem Superioritätsdünkel in Europa ein Glaubwürdigkeitsproblem hatte, gegen das er offensiv angehen musste. Daher enthielt seine Historia an einer Unzahl von Stellen Hinweise auf die Fundierung des eigenen Wissens, auf vorhandene Dokumente, überlieferte Schriften und nachvollziehbare Zeugnisse, die seine Ar-

31 Clavijero, Francisco Javier: Historia antigua de México, S. $103 \mathrm{f}$. 
gumentation stützen konnten. Selbstverständlich wusste Clavijero auch, dass er als Mitglied der Societas Jesu in den Augen vieler europäischer Aufklärer als Kleriker nicht glaubwürdig war, auch wenn viele der europäischen ,Philosophes pro forma“ ebenfalls als „Abbés“ wie Guillaume-Thomas Raynal oder Cornelius de Pauw beschäftigt waren. All dies erzeugte eine bisweilen defensive Argumentation in der Historia, wie wir sie in der obigen Passage antreffen und wie sie ohne dieses Vorwissen um die diskursive Stellung von Clavijeros Schrift nicht wirklich begriffen werden kann. Denn Clavijero stand in der Debatte, im internationalen Disput um die Neue Welt und damit in den Auseinandersetzungen zwischen einer europäischen und einer amerikanischen Aufklärung von Beginn an unter Druck.

In der obigen Passage kann es keinen Zweifel an der Strategie des neuspanischen Jesuiten geben: Clavijero bemühte sich redlich, alle Funktionen und Aufgaben innerhalb des Staatswesens, von der Spitze, dem König, bis hinunter zu den Professoren oder Lehrern an der Akademie für seine Leserschaft in ein europäisches Sinnsystem zu übersetzen und damit natürlich auch für Europa zu ,aktualisieren'. Nur allzu deutlich schimmert die Idealvorstellung von einem guten Herrscher und seinem „buen gobierno“ in diesen Schilderungen der Herrschaft Nezahualcóyotls durch. Der Dichter-Fürst erscheint als aufgeklärter Monarch im Stile des 18. Jahrhunderts.

Doch Clavijero war bezüglich des Staatswesens von Tezcoco keineswegs auf seine eigene Phantasie angewiesen. Denn es gab gerade in diesem Bereich eine Vielzahl von Quellen und Dokumenten, die - neben den Gedichten Nezahualcóyotls selbst - die dessen Bedeutung und die mit ihm verbundene Regierungsform belegen konnten. So ergab sich gerade bei der Behandlung der Herrschaft von Nezahualcóyotl der größte Gegensatz zu den Behauptungen eines de Pauw, eines Robertson oder eines Raynal, die den sogenannten Indianern jegliche kulturelle Entwicklung absprachen und sie ausschließlich im Lichte ihrer inhärenten Schwäche und Inferiorität präsentierten. Denn das von Clavijero geschilderte Staatswesen war höchst vernünftig konzipiert und mit Institutionen ausgestattet, welche geradezu einer abendländischen Rationalität entsprungen zu sein schienen.

Francisco Javier Clavijero forderte ganz bewusst für die altamerikanischen Kulturen und deren Zivilisationen eine Position und Stellung ein, welche zumindest mit jener der griechischen Stadtstaaten oder den Anfängen des Römischen Reiches vergleichbar war. Darauf macht dieses Zitat und auch der Hinweis auf den Aufbau einer Akademie, der Anlage von Parks und Forsten, der Einrichtung von Schulen und Bildungsstätten im Kontext einer umsichtigen Politik und einer großen Vaterlandsliebe aufmerksam - allesamt Zeugnisse einer zivilisatorischen Entwicklung, wie sie in der mediterranen Antike entstanden und noch im 18. Jahrhundert sehr geschätzt wurden. 
Wüssten wir nicht, von welchem Kontinent, von welchem Zeitalter und von welchem Herrscher gerade die Rede ist, wir könnten uns in die Epoche von Friedrich dem Großen versetzt fühlen, der ebenfalls die Arbeit der Akademie förderte, selbst musisch aktiv war und sowohl Bildungsinstanzen wie die Verwaltung seines aufblühenden Staatswesens unterstützte. Dass er für zahlreiche Bauwerke innerhalb wie außerhalb der Stadt, aber auch für großzügige Parks, Forste und Wälder verantwortlich zeichnete, können Sie noch heute in und um Potsdam eindrucksvoll erleben. Größer könnte der Unterschied zum indolenten Indianerbild eines de Pauw, Robertson oder Raynal nicht mehr sein. Hier ist das Bild der Aufklärung nach Amerika übergewechselt und wird in eine Vergangenheit zurückprojiziert, die nun zur eigenen Vergangenheit der Kreolen geworden ist. Und über eine solche Welt brach der Sturm der Conquista, brach die Unterjochung durch die Soldateska der Spanier herein! Dies ist eine literarische und künstlerische Konfiguration, die kein Geringerer als Friedrich der Große - wie wir gleich sehen werden - in Form einer Oper durchspielen sollte; eine Oper, von der Clavijero jedoch wohl nichts wissen konnte.

Am Ende des obigen Zitats verweist letzterer auf die „Disertaciones“; dabei handelt es sich um insgesamt neun längere Texte und Untersuchungen, die seiner Historia antigua de México angehängt wurden und die unterschiedlichsten Themenbereiche abdecken. Das vom neuspanischen Jesuiten bearbeitete Themenspektrum reicht von den Hauptepochen der amerikanischen Geschichte bis zu den in Amerika vorkommenden Tieren, von der Religion der Indianer bis hin zu Herkunft und Verbreitung der Syphilis nach dem damaligen Kenntnisstand. Denn Clavijero hatte sehr wohl verstanden, welche Rolle den Epidemien bei der Entstehung einer vernetzteren Welt zukam.

Ich möchte sie zu diesen Themen allerdings nicht näher unterrichten, denn wir haben uns zum Teil ja bereits damit beschäftigt. Aber ich möchte Ihnen doch noch einige wenige Überlegungen Clavijeros aus diesen nachgestellten „Disertaciones“ seiner Historia antigua de México vorstellen, damit Sie sich ein Bild davon machen können, dass die Aufklärung nicht nur einfach in die Vergangenheit projiziert wurde, sondern in Clavijeros Werk in Amerika voranschreitet und sich entwickelt.

Ich habe dafür die fünfte Dissertation ausgesucht, die sich in einem ersten Teil zunächst mit der körperlichen Beschaffenheit der indigenen Bevölkerung auseinandersetzt, bevor sich der Seelenhirt Clavijero - nicht umsonst ein Jesuit - mit den indianischen Seelen in einem zweiten Teil beschäftigt. Ich kann auf diese spezielle Frage an dieser Stelle unserer Vorlesung jedoch aus Zeitgründen nicht eingehen, so spannend die Erörterung dieser Problematik auch wäre. Doch möchte ich Ihnen gerne zeigen, dass wir mit Montesquieu und seinen Lettres persanes nicht nur die Geburt eines anthropologischen oder ethno- 
graphischen Blickes auf das Eigene in Europa beobachten können, sondern auch eine Art Geburt des kritischen amerikanischen Blickes auf Europa.

Man könnte diesbezüglich leicht ironisch von einer ,Gegenaufklärung‘ sprechen, welche sich gegen die europäische Aufklärung und weit mehr noch gegen bestimmte europäische Aufklärer richtet. Denn in mancherlei Hinsicht war die europäische Aufklärung mit Blick auf die Amerikas weder Aufklärung noch Verklärung, sondern schlicht die Verbreitung europäischer Vorurteile: ein Diskurs bar jeglicher Selbstkritik. In dieser unsere Auseinandersetzung mit Clavijero abschließenden Passage ist die Umkehrung dieses Blickes spürbar. Es handelt sich um einen ,Gegen-Blick', der noch im 20. Jahrhundert - wie wir bereits sahen - für die Europäer weithin unerträglich war. Dieser Blick aber wird von Clavijero in diesen beiden Schlussabschnitten seiner „quinta disertación“ bereits um 1780 deutlich eingefordert:

Aber jedwedem Amerikaner von mittlerer Intelligenz und einiger Bildung, der es in derselben Münze diesen Schriftstellern (wie wir anderswo über einen Philosophen aus Guinea gesagt haben) heimzahlen wollte, fiele es leicht, ein Werk mit diesem Titel zu verfassen: Philosophische Untersuchungen über die Bewohner des Alten Kontinents. Indem man dieselbe Methode von de Pauw befolgte, würde man das wiederaufnehmen, was über sterile Länder der Alten Welt geschrieben wurde, über unerreichbare Berge, über sumpfige Ebenen, undurchdringliche Wälder, sandige Wüsten und schlechte Klimate, eklige und schädliche Reptilien und Insekten, Schlangen, Schädlinge, Skorpione, Termiten, Frösche, Tausendfüßler, Kakerlaken, Wanzen und Läuse, ungleiche, kleinwüchsige, verquere und furchtsame Vierfüßler, über degenerierte Menschen von hässlicher Farbe, unausgewogener Statur, deformierten Gesichtszügen, schlechter Haltung, von gedrückter Stimmung, verdunkelter Einsicht und grausamem Äußeren.

Wenn es dann zum Artikel über die Laster käme: Welche unendliche Vervielfachung von Materialien gäbe es hier nicht für ein solches Werk! Welche Exemplare von Niedertracht, Perfidie, Grausamkeit, Aberglaube und Auflösung gäbe es hier! Welche Exzesse in jedweder Art von Lastern! Allein die Geschichte der Römer, der berühmtesten Nation der Alten Welt, würde einem eine unglaubliche Vielzahl der horrendesten Übeltaten liefern. Man müsste einräumen, dass derartige Defekte und Laster weder in allen Ländern noch bei allen Bewohnern des Alten Kontinents verbreitet waren; doch zählte all dieses nicht, denn man würde entlang desselben Modells von de Pauw schreiben und sich derselben Logik bedienen. Ein solches Werk wäre ohne Zweifel weitaus beliebter und glaubwürdiger noch als dasjenige von de Pauw, denn wenn dieser Philosoph nicht gegen Amerika und die Amerikaner nur aus europäischen Autoren zitiert, so würde im Gegensatz dazu der amerikanische Schriftsteller für sein kurioses Werk ausschließlich Autoren benutzen, die von diesem selben Kontinente stammen, gegen den sich diese Schrift richtete. ${ }^{32}$

In dieser hochpolemischen Passage kann es Francisco Javier Clavijero nicht unterlassen, erneut auf den Philosophen aus Guinea hinzuweisen und eine Ver-

32 Clavijero, Francisco Javier: Historia antigua de México, Quinta Disertación, S. 524. 
bindung zu der bereits früher in unserer Vorlesung zitierten Passage aus seiner Historia antigua de México herzustellen. Es ist offenkundig, dass der neuspanische Jesuit Kunde haben musste von jenem Anton Wilhelm Amo, der zum Zeitpunkt des Erscheinens der Historia längst Deutschland wieder verlassen hatte, aber sich im gesamten 18. Jahrhundert einer großen nationalen wie internationalen Bekanntheit erfreute. Darum wiegt, so will mir scheinen, das Schweigen der deutschen Philosophen des 18. Jahrhunderts wie Immanuel Kant auch so schwer, gemahnt es doch an ein Totschweigen des in Deutschland akademisch sozialisierten und durchaus erfolgreichen schwarzen Philosophen.

In diesem Zusammenhang ist unklar, wieviel Clavijero von Amos Philosophie bekannt war. Aber es dürfte unstrittig sein, dass er ihn als ein Beispiel für eine Perspektivik auf Europa und die europäische Philosophie der Aufklärung wählte, welche ebenso wie die seine Europa nicht allein von einer inneren, sondern zugleich von einer äußeren Perspektivik aus mit einer der Aufklärung würdigen kritischen Haltung betrachtete. Der „Philosoph aus Guinea“, wie sich Amo bisweilen selbst nannte, war für den in Neuspanien geborenen Philosophen sehr wohl ein Begriff.

In der obigen Passage wurde ein Buchprojekt skizziert, das auf den ersten Blick als völlig undurchführbar erscheinen könnte. Denn fürwahr: Welch ein Buchprojekt! Doch wir könnten hinzufügen: Es wurde wenige Jahrzehnte später von einem Landsmann Clavijeros und einem weiteren Mann der Kirche verwirklicht - freilich nicht von einem Jesuiten, sondern einem Dominikaner. Es war kein Geringerer als Fray Servando Teresa de Mier, eine der großen Figuren der mexikanischen und hispanoamerikanischen Unabhängigkeitsrevolution, mit dessen späteren Schriften wir uns bereits in unserer Vorlesung zum 19. Jahrhundert beschäftigt haben, war er doch auch der Verfasser der wohl ersten Übersetzung von Chateaubriands romantischem Schlüsseltext Atala ins Spanische. ${ }^{33}$

Fray Servando Teresa de Mier y Guerra ließ in seinem „Reisebericht eines Amerikaners“ in Europa keinen der Ratschläge Clavijeros aus, eine Art umgedrehte Sichtweise von Cornelius de Pauws Recherches philosophiques sur les américains zu verfassen. Darum sollen ihm auch in dieser Vorlesung noch einige Seiten gewidmet sein. In der Tat finden sich in seinem Reisebericht degenerierte Ureinwohner in Europa zuhauf, wie schon ein erster Blick auf die Einwohner der spanischen Hauptstadt, also der Hauptstadt des Kolonialreiches verdeutlichen sollte. Der neuspanische Dominikanermönch machte sich einen Spaß daraus, die großen Eroberer und Herrscher über die Neue Welt als schwäch-

33 Vgl. hierzu den vierten Band der Reihe „Aula“ in Ette, Ottmar: Romantik zwischen zwei Welten, S. $153 \mathrm{ff}$. 
liche, missgestaltete, dümmliche und übelmeinende Menschlein zu porträtieren, von denen man sich nun aus amerikanischer Sicht endgültig trennen müsse. Er verwirklichte mit großer Lust - ob bewusst oder unbewusst, das bedürfte weiterer Forschungen und muss hier offen bleiben - jenes literarische Schreibprogramm, das Francisco Javier Clavijero in seiner fünften „Disertación“ Jahrzehnte zuvor als Verhohnepiepelung de Pauws skizziert hatte.

In dem oben aufgezeigten Sinne sehen wir mithin eine Seite der europäischen Aufklärung, die bislang in Europa unbeleuchtet und damit unaufgeklärt blieb: die radikale Aussperrung eines Teiles der Menschheit durch europäische Aufklärungsphilosophen unter dem Vorwand einer Universalisierung von Werten, die wir selbst noch bei Georg Wilhelm Friedrich Hegel, bei Karl Marx oder bei Friedrich Engels wiederfinden können. Es wäre ein Leichtes, diese Spuren bis ins 20. und 21. Jahrhundert nachzuzeichnen. Doch möchte ich Ihnen zumindest an dieser Stelle einmal aufgezeigt haben, in welch erstaunlichem Maße das 18. Jahrhundert - wenn wir das programmatische Bild von der „Aufklärung zwischen zwei Welten“ ernstnehmen - Möglichkeiten zutage förderte, das Eigene in neue Beziehungen einzubeziehen und gemeinsam mit der Entdeckung des Anderen auch eine Entdeckung des Eigenen hervorzubringen. Dabei ist diese ,Entdeckung des Eigenen“ nicht als ein Sich-Abschließen über einem wie auch immer sich konstituierenden ,Eigenen' zu denken, sondern als eine Weitung und Erweiterung, deren Epistemologie zu diesem Zeitpunkt noch nicht ausgedacht war.

Denn unbestreitbar ist: Clavijeros Historia antigua de México ist eine Ethnographie und Kulturgeschichte, die auf die zeitgenössischen Diskussionen des 18. Jahrhunderts bezogen ist und Teil hat an jenen aufklärerischen Auseinandersetzungen, die das „Siglo de las Luces“, das „Siècle des Lumières“ mit Blick auf die welthistorischen Veränderungen Im Umfeld der zweiten Phase beschleunigter Globalisierung entfesselte. Die Geschichte des Altamerikanischen als Amerikanischem ist zugleich die Konstruktion eines Eigenen, das dann auch wenige Jahre später den amerikanischen Kreolen dazu dienen sollte, eine Frontstellung gegenüber dem Anderen, den Spaniern aufzubauen, obwohl diese den Kreolen doch genealogisch sehr viel näher standen als der indigenen Bevölkerung. Doch die Konstruktion von Vergangenheit geht mit der von Zukunft Hand in Hand.

Die indigenen Bevölkerungen wiederum sollten in diesem Spiel der kreolischen Eliten nicht zum Anderen im Eigenen, sondern erneut - und auf vielleicht noch stringentere, radikalere Weise - ganz aus dem Eigenen ausgeschlossen werden. Aber auch dies ist eine Geschichte, die im 19. Jahrhundert ihre eigentliche Fortsetzung in der politischen Unabhängigkeit und ihren Höhepunkt in der Konstituierung der verschiedenen Nationalstaaten des künftigen Lateinamerika 
erreichen sollte; eine Geschichte, an der auch wir noch am Beginn des 21. Jahrhunderts partizipieren. So ist Clavijeros Projekt einer anderen, einer altamerikanischen Antike und damit einer amerikanischen Moderne weit davon entfernt, in diesem Sinne eingelöst oder erlöst worden zu sein. Denn von einer politischen Gleichstellung der indigenen Bevölkerungen kann nicht im Ansatz die Rede sein. Das eigentlich Spannende an dieser Geschichte ist die Tatsache, dass in dieser Aufklärung zwischen zwei Welten nun die Werte der europäischen Aufklärung als immerhin ,universalgültige‘ Werte nun gegen diese europäische Aufklärung oder doch einen Teil ihres vorgeblichen Universalismus - und keinen geringen Teil! - in Stellung gebracht wurden und daraus eine kulturelle Problematik entstand, die bis heute nicht ansatzweise aufgearbeitet wurde.

Doch ich hatte Ihnen die Verfolgung einer Linie versprochen, die ganz in unsere räumliche Nähe führt, nach Schloss Sanssouci, und keinen Geringeren als Friedrich den Großen in den Zeugenstand ruft. Wenden wir uns also Fragestellungen zu, die erneut dem Zusammenhang zwischen Alter und Neuer Welt gelten. Wir stellen Friedrich II. einen Autor an die Seite, dessen Name längst wieder in der Dunkelheit versunken ist, der aber - wie wir auch bei Clavijero sahen - einer der zentralen Autoren des 18. Jahrhunderts war und dessen Name in der sogenannten Berliner Debatte um die Neue Welt hell leuchtete. Es war eine weltweite Debatte, die sich gleichsam vor unserer Haustür abspielte und die uns zeigt, dass wir hier an der Universität Potsdam in den wunderbaren historischen Gebäuden, die uns direkt mit dem 18. Jahrhundert verbinden, hautnah dran sind an jenem Geschehen, das wir nun aus einer anderen Perspektive diskutieren wollen. Wir befinden uns nämlich unweit jener Gebäude und Wandelhallen, in denen sich einst Voltaire und Friedrich der Große oder Cornelius de Pauw, Guillaume-Thomas Raynal und Giacomo Casanova aufhielten. Vielleicht spüren Sie noch etwas von jenem Geist, wenn Sie unter den Kolonnaden am Neuen Palais lustwandeln ... 